Rechtspolitisches Forum Legal Policy Forum

31

Thomas von Danwitz

Wege zu besserer Gesetzgebung in Europa 
Das Institut für Rechtspolitik an der Universität Trier hat die wissenschaftliche Forschung und Beratung auf Gebieten der Rechtspolitik sowie die systematische Erfassung wesentlicher rechtspolitischer Themen im In- und Ausland zur Aufgabe. Es wurde im Januar 2000 gegründet.

Das Rechtspolitische Forum veröffentlicht Ansätze und Ergebnisse national wie international orientierter rechtspolitischer Forschung und mag als Quelle für weitere Anregungen und Entwicklungen auf diesem Gebiet dienen. Die in den Beiträgen enthaltenen Darstellungen und Ansichten sind solche des Verfassers und entsprechen nicht notwendig Ansichten des Instituts für Rechtspolitik. 
Das Streben nach besserer Gesetzgebung ist ebenso alt wie die Klage über die unzureichende Qualität und die schier haltlose Flut der erlassenen Vorschriften. Symptomatisch für die vielfältigen Defizite heutiger Gesetzgebung auf nationaler Ebene sind systematische Unstimmigkeiten und oftmals kaum auflösbare innere Widersprüche von Gesetzen. Die Probleme und Herausforderungen, die sich aus der europäischen Rechtsetzung für die Verwirklichung der Zielsetzung einer guten Gesetzgebung ergeben, sind bis dato weitgehend unbehandelt. Der Beitrag befasst sich mit den Schwierigkeiten der gemeinschaftlichen Rechtsetzung, der Mitwirkung der Mitgliedstaaten und der Umsetzung in nationales Recht.

Prof. Dr. Thomas von Danwitz, Jahrgang 1962, ist seit 2003 Professor für Öffentliches Recht und Europarecht, insb. Medien- und Kommunikationsrecht an der Universität zu Köln. Zuvor war er von 1996 bis 2002 an der Ruhr-Universität Bochum als Professor für Europarecht tätig. Seine Forschungs- und Veröffentlichungsschwerpunkte liegen im Medien-, Umwelt- und Europarecht. 
Der Beitrag ist der um Literaturhinweise ergänzte Abdruck des Vortrages, den der Verfasser am 12. Juli 2005 gehalten hat. 


\title{
WEGE ZU BESSERER GESETZGEBUNG IN EUROPA
}

\author{
PROF. DR. THOMAS VON DANWITZ \\ Universität zu Köln
}

\section{Problemstellung}

Das Streben nach besserer Gesetzgebung ist ebenso alt wie die Klage über die unzureichende Qualität und die schier haltlose Flut der erlassenen Vorschriften1. Unwillkürlich erinnert die ernsthafte Fachdiskussion mitunter an den uralten Witz über die verständnisheischende Verteidigung des liebenswerten Ganoven, dass ein Einzelner gegen die über 350 Paragraphen des Strafgesetzbuches doch nichts ausrichten könne. Jedoch hat sich die Dramatik der Situation in den vergangenen zwanzig Jahren erheblich verschärft. Sie lässt es nicht mehr zu, das Thema resignativ oder achselzuckend als Problem der politischen Entscheidungsprozesse von der staatsrechtlichen Agenda zu nehmen2. Denn die handwerkliche, legistische Qualität der Rechtsetzung nimmt beständig ab, ja die Defizite nehmen ein solches Ausmaß an, dass die gesetzliche Steuerung der Verwaltung beeinträchtigt wird und die Gesetzesbefolgungsbereitschaft der Bürger auf breiter Front leidet3. Symptomatisch für die vielfältigen Defizite heutiger Gesetzgebung sind systematische Unstimmigkeiten und oftmals kaum auflösbare innere Widersprüche von Gesetzen, zumal wenn sie im Vermittlungs-

1 Dazu mit Nachweisen schon Klaus Stern, Das Staatsrecht der Bundesrepublik Deutschland, Band II, 1980, S. 639 ff.

2 Zur aktuellen Diskussion aus nationaler Sicht vgl. insbesondere Peter Blum, Wege zur besseren Gesetzgebung - sachverständige Beratung, Begründung, Folgeabschätzung und Wirkungskontrolle, Gutachten I zum 65. DJT, 2004; ferner die Begleitaufsätze zum DJT 2004 von Jörg Ennuschat, Wege zur besseren Gesetzgebung, DVBI. 2004, S. 986 ff.; Helmuth Schulze-Fielitz, Wege, Umwege oder Holzwege zu besserer Gesetzgebung, JZ 2004, S. 862 ff.; Wolfgang Thierse, Wege zu besserer Gesetzgebung, NJW 2005, S. 153 ff.; außerdem etwa Konrad Redeker, Wege zu besserer Gesetzgebung, ZRP 2004, S. 160 ff.; ders., Auf der Suche nach besserer Gesetzgebung, NJW 2002, S. 2756 ff.

Dazu Redeker (o. Fußn.2), NJW 2002, S. 2756 f. 
verfahren erlassen werden. Ebenso problematisch ist der Verzicht der Gesetzessprache auf dogmatisch geprägte und inhaltlich bestimmte Rechtsbegriffe 4 , der mit einem zunehmenden Ausweichen auf ökonomische und soziologische Begriffswelten oder die Alltagssprache einhergeht, die keine hinreichend bestimmten Begriffsinhalte aufweisen und dem Gesetz eine oftmals beträchtliche Vieldeutigkeit verleihen. Die festzustellende Intransparenz gesetzlicher Bestimmungen und die für Außenstehende oftmals nicht nachvollziehbare Geschwindigkeit, mit der Gesetze, teilweise vor dem Zeitpunkt ihres Inkrafttretens, geändert werden, sprechen den Anforderungen von Rechtssicherheit und Voraussehbarkeit oftmals Hohn.

Für die moderne Gesetzgebung ist überdies geradezu kennzeichnend geworden, dass sie immer stärker politisch-symbolische Ziele verfolgt und immer weniger darauf angelegt ist, der Verwaltung demokratisch legitimierte Handlungsermächtigungen zu erteilen, sie an rechtsstaatliche Voraussetzungen zu binden und einen juristisch handhabbaren Maßstab für die gerichtliche Kontrolle und Streitentscheidung festzulegen. Das oftmals selbstverständlich vorgetragene Bekenntnis der politischen Klasse zur Notwendigkeit dieser symbolischen Gesetzgebung bildet aber fraglos einen Tiefpunkt rechtskultureller, aber vor allem demokratischer Verirrung. Eine Demokratie, in der Gesetze nicht mehr auf Vollzug ausgerichtet werden und die Vieldeutigkeit ihrer Rechtsbegriffe es weitgehend dem Richter überlässt, „etwas Vernünftiges daraus zu machen", erweist sich als ein System organisierter Unverantwortlichkeit, das seine Legitimation verspielt und der Politikverdrossenheit Tür und Tor öffnet.

\section{Zum Stand der Diskussion}

Dieser Befund ist ebenso schwerwiegend wie eindeutig, so dass trotz aller Divergenzen in der Bewertung einzelner Phänomene weitgehende Einigkeit in der Feststellung eines dringlichen Handlungsbedarfs besteht. Ebenso übereinstimmend und berechtigt

4 Siehe Paul Kirchhof, Sprachstil und System als Geltungsbedingung des Gesetzes, NJW 2002, S. 2760 ff.; eingehend Hans Schneider, Gesetzgebung, 3. Auflage 2002, § 13. 
wurde auf dem 65. Deutschen Juristentag im vergangenen Jahr jedoch darauf hingewiesen, wie unangemessen es wäre, diese Fehlentwicklungen zur Grundlage für eine allgemeine Gesetzgebungskritik oder eine pauschale Politikerschelte zu machen5. Insbesondere gilt es der Versuchung zu widerstehen, die Gesetzgebung technokratisch zu usurpieren und ihre genuin politische Natur negieren zu wollen. Es geht vielmehr umgekehrt darum, die politische Handlungs- und Entscheidungsfreiheit zu stärken, indem ihre demokratischen Gestaltungsoptionen transparent gemacht und die Steuerungsleistungen moderner Gesetzgebung gegenüber Verwaltung und Gerichten verbessert werden6. Im Hinblick auf die Diskussionen auf dem letztjährigen Juristentag und die öffentlichen Reaktionen kann man in diesem Sinne mit Befriedigung feststellen, dass weitgehende Übereinstimmung in der Problemanalyse und in der Schlussfolgerung besteht, dass ein dringlicher Handlungsbedarf unabweisbar geworden ist. Daher hat sich der Schwerpunkt in dieser Diskussion auf die Suche nach probaten Therapiemöglichkeiten verlagert. In den Diskussionen auf dem 65. DJT ging die Meinungsbildung eindeutig zu einer behutsamen Fortentwicklung des Gesetzgebungsverfahrensrechts durch Einfügung institutioneller und verfahrensrechtlicher Sicherungen. Die Schaffung eines organisatorisch unabhängigen Gesetzgebungs- und Beratungsdienstes beim Deutschen Bundestag nach dem Vorbild Niedersachsens7, die Aufwertung der regierungsseitigen Rechtsförmlichkeitsprüfung durch eine Gewährleistung ausreichender Bearbeitungsfristen und die ausdrückliche Verantwortungsübernahme der Regierung für die legistische Qualität eines Gesetzentwurfs durch entsprechendes Testat8, eine Begründungs- bzw. Erklärungsnotwendigkeit für die im Vermittlungsausschuss erzielten Beratungs-

5 So der übereinstimmende Tenor zahlreicher Stellungnahmen auf dem 65. DJT 2004, vgl. exemplarisch die Referate von Wolfgang Thierse und Jochen Diekmann, in: Verhandlungen zum 65. DJT 2004, Band II/1, S. S $9 \mathrm{ff}$. und S $19 \mathrm{ff}$.

6 Vgl. etwa Udo Di Fabio, Referat zum 65. DJT, 2004, S. S $47 \mathrm{ff}$.

7 Dazu eingehend Blum (o. Fußn. 2), Gutachten I S. 139 ff.; vgl. auch Bernd Brunn, Möglichkeiten einer Rechtsförmlichkeitsprüfung im parlamentarischen Verfahren, ZRP 2004, S. $79 \mathrm{ff}$.

Di Fabio (o. Fußn. 6), S. S 56. 
ergebnisse9 sowie die verpflichtende Durchführung einer retrospektiven Gesetzesfolgenabschätzung10 standen insoweit im Vordergrund der Überlegungen.

\section{Wege zur besseren Gesetzgebung in Europa}

Während diese fachliche Diskussion der innerstaatlichen Problemstellung einen gewissen Sättigungsgrad erreicht hat und weniger nach fachlicher Verfeinerung als nach rechtspolitischer Akzeptanz und Verwirklichung verlangt, sind die Probleme und Herausforderungen bis dato noch weitgehend unbehandelt, die sich aus der europäischen Rechtsetzung für die Verwirklichung der Zielsetzung einer guten Gesetzgebung ergeben11. Dieser Umstand ist besonders bedauerlich, da wir seit einer entsprechenden Erklärung des seinerzeitigen Kommissionspräsidenten Jacques Delors davon ausgehen, dass die Hälfte aller in den Mitgliedstaaten geltenden Gesetze auf gemeinschaftlicher Rechtsetzung beruhen und sogar $80 \%$ des geltenden Wirtschafts- und Sozialrechts originär gemeinschaftsrechtlichen Ursprungs sind12. Für die gemeinschaftliche Rechtsetzung stehen wir in der Ursachenforschung und der rechtswissenschaftlichen Problemanalyse noch ganz am Anfang. Insbesondere ist zu bedenken, dass die Komplexität der Problemlage und das Fehlen empirisch abgesicherter Analysen den Betrachter in diesem ohnehin wertungsbehafteten Umfeld auf eigene Erfahrungen und Anschauungen zurück werfen, die notwendigerweise nur einen kleinen und zudem subjektiv geprägten Ausschnitt

9 So bereits Konrad Redeker/Ulrich Karpenstein, Über Nutzen und Notwendigkeiten, Gesetze zu begründen, NJW 2001, S. 2825 (2826); eingehend zu verfassungsrechtlichen und verfassungspolitischen Fragen der Begründung von Gesetzen Blum (o. Fußn. 2), Gutachten I S. 123 ff. sowie die Monographien von Uwe Kischel, Die Begründung, 2002, und Panagiotis Skouris, Die Begründung von Rechtsnormen, 2002.

10 Zur Folgenbewertung zuletzt Ulrich Karpen, Nachträgliche Wirkungskontrolle von Gesetzen, Referat zum 65. DJT, S. S 33 ff.

11 Siehe aber bereits Hans-Werner Rengeling, Überlegungen zur Qualität des Gemeinschaftsrechts, in: ders. (Hrsg.), Handbuch zum europäischen und deutschen Umweltrecht, Band II/2, 2. Auflage 2002, § 92.

12 Rede im Europäischen Parlament am 4. Juli 1988, Bulletin EG 1988, Nr. 7/8, S. 124. 
aus der Problemfülle erfassen können. Insofern kann es nur darum gehen, einen Anfang zu machen.

Es liegt auf der Hand, dass diese Fragestellung zwei unterschiedliche, wenngleich miteinander verbundene Problemkreise aufweist: die Ebene der gemeinschaftlichen Rechtsetzung einerseits und die Ebene der mitgliedstaatlichen Vorbereitung und Mitwirkung andererseits sowie schließlich die Umsetzung gemeinschaftsrechtlicher Vorgaben13. Ich wende mich zunächst der europäischen Ebene $\mathrm{zu}$.

\section{Die Ebene gemeinschaftlicher Rechtsetzung}

Unterzieht man die institutionellen Rahmenbedingungen der gemeinschaftlichen Rechtsetzung einer näheren Betrachtung, so fallen zunächst die erheblichen Unterschiede ins Auge, die gegenüber den parlamentarischen Regierungssystemen der Mitgliedstaaten bestehen. Das trianguläre Rechtsetzungssystem der Gemeinschaft verbindet zentripetale mit zentrifugalen Kräften zu einer Gewaltenverschränkung sui generis. Die vielfältigen checks and balances, die namentlich im Mitentscheidungsverfahren nach Art. 251 EG ausgeprägt worden sind, bedingen fraglos, dass sich die gemeinschaftliche Rechtsetzung in besonderem Maße kompromisshaft gestaltet und oftmals nur auf dem kleinsten gemeinsamen Nenner erfolgt, der nicht selten durch dilatorische Formelkompromisse gebildet wird. Zwar sorgen das Initiativmonopol der Kommission und ihre daraus resultierende, starke Stellung im weiteren Rechtsetzungsverfahren im Allgemeinen dafür, dass die Rechtsetzungsvorschläge eine in sich stimmige Konzeption enthalten und dem Idealbild der Rechtsetzung „aus einem Guss“ durchaus nahe kommen können. Oft genug wird die Ausgangskonzeption der Kommission in den Beratungen von Rat und Parlament jedoch bis zur Unkenntlichkeit verändert oder durch die Modifikation von Einzelpunkten so weitgehend zerpflückt, dass die endgültigen Regelungen eigentlich nur noch in formaler Hinsicht auf den ursprüngli-

13 Siehe dazu für Frankreich den Circulaire relative à la procédure de transposition en droit interne des directives et décisions-cadres négociées dans le cadre des institutions européennes vom 27. September 2004, J.O n² 230 vom 2. Oktober 2004, S. 16920. 
chen Kommissionsvorschlag zurückgeführt werden können14. Denkt man zudem an die auf Grund integrationspolitischer Zurückhaltung wohl etablierten Regelungsformen der Mindest- und vor allem der optionalen Harmonisierung15, wird die Größenordnung des Problems erst in vollem Umfang erkennbar.

Dennoch ist es wenig hilfreich und offenbart zudem ein unzureichendes Verständnis von der Notwendigkeit demokratischer Legitimation auf europäischer Ebene, wenn man sich damit begnügt, die "Schuld“ für Unstimmigkeiten und regelungstechnische Widersprüche im gemeinschaftlichen Sekundärrecht den im Rat versammelten Mitgliedstaaten, ihrer unzureichenden Integrationsbereitschaft oder dem Europäischen Parlament zuzuweisen. Es handelt sich dabei vielmehr um notwendige Gegengewichte zum Initiativmonopol der Kommission, die als institutionelle Rahmenbedingungen hinzunehmen sind. Selbst wenn man bedenkt, welche Belastung ein auf annährend 30 Mitglieder angewachsener Rat für die Kohärenz der Rechtsetzung bedeutet, und auch die Augen vor dem mitunter erstaunlichen Einfluss von organisierten Interessengruppen auf die Tätigkeit vor allem von Kommission und Parlament nicht verschließt16, bedingt eine solch realistische Sicht der Dinge keineswegs eine Kapitulation vor dem Wildwuchs der Unkultur, die auch in die gemeinschaftliche Rechtsetzung Eingang gefunden hat.

14 Insoweit kann beispielhaft auf die überaus zahlreichen Änderungsvorschläge verwiesen werden, die dem Europäischen Parlament im Hinblick auf den Kommissionsvorschlag für eine Verordnung des Europäischen Parlaments und des Rates über nährwert- und gesundheitsbezogene Angaben über Lebensmittel unterbreitet worden sind. Siehe dazu den Bericht von Adriana Poli Bortone vom 2. Mai 2005, Parlamentsdokument-Nr. A6-0128/2005.

15 Siehe dazu Thomas von Danwitz, in: Dauses (Hrsg.), Handbuch des europäischen Wirtschaftsrechts, Bd. 1, B. II. 5., Rn. 84 ff. (Loseblatt; Stand der Bearbeitung: März 2002).

16 Siehe dazu - mit Blick auf den Vorschlag für eine Richtlinie des Europäischen Parlaments und des Rates über die Patentierbarkeit computerimplementierter Erfindungen [KOM (2002) 92 endg.] - den Bericht von Hendrik Kafsack/Werner Mussler, Die Schlacht ums Patent, FAZ Nr. 152 vom 4. Juli 2005, S. 15. 


\section{a) Initiativen und Maßnahmen zur Verbesserung der Gesetzge- bungsqualität auf Gemeinschaftsebene}

An Initiativen und Maßnahmen zur Verbesserung der Gesetzgebungsqualität auf Gemeinschaftsebene hat es freilich schon in der Vergangenheit nicht gefehlt und fehlt es auch heute nicht. Erst jüngst hat das Thema eine besondere Akzentuierung erfahren, indem es von der britischen Präsidentschaft zu einer prioritären Aufgabenstellung erklärt wurde17. Besonders hervorzuheben sind insoweit eine Reihe von interinstitutionellen Vereinbarungen18. In diesen haben sich die Gesetzgebungsorgane der Gemeinschaft im Sinne einer ausdrücklichen Bekräftigung ohnehin bereits bestehender Rechtspflichten - darauf verständigt, im Rahmen ihrer Tätigkeit die Grundsätze der demokratischen Legitimität, der Subsidiarität und der Verhältnismäßigkeit sowie der Rechtssicherheit zu beachten. Bekräftigt wird ferner die Notwendigkeit einer einfachen, klaren und kohärenten Formulierung von Gesetzestexten und eines Höchstmaßes an Transparenz im Rechtssetzungsverfahren19. Von Seiten der Kommission wurde überdies im Jahre 2002 ein Aktionsplan zur „Vereinfachung und Verbesserung des Regelungsumfeldes" vorgelegt20, an den sie nunmehr mit ihrer Mitteilung über „Bessere Rechtsetzung für Wachstum und Arbeitsplätze in der Europäischen Union" angeknüpft hat21. In dieser Mitteilung nennt die Kommission im Kern drei Maßnahmen, von denen sie sich eine verbesserte Qualität der Gesetzgebung auf Gemeinschaftsebene

17 Siehe dazu „Priorities for the UK Presidency of the EU 2005”, einsehbar unter http://www.eu2005.gov.uk/servlet/Front?pagename=OpenMarket/ Xcelerate/ShowPage\&c=Page\&cid $=1107293521089$.

18 Interinstitutionelle Vereinbarung von Europäischem Parlament, Rat und Kommission vom 22. Dezember 1998 über Gemeinsame Leitlinien für die redaktionelle Qualität der gemeinschaftlichen Rechtsvorschriften, ABI. EG Nr. C 73 vom 17. März 1999, S. 1 ff.; Interinstitutionelle Vereinbarung vom 28. November 2001 über die systematischere Neufassung von Rechtsakten, ABI. EG Nr. C 77 vom 28. März 2002, S. 1 ff.; Interinstitutionelle Vereinbarung „Bessere Rechtsetzung“, ABI. EG Nr. C 321 vom 31. Dezember 2003, S. 1 ff.

19 Siehe etwa Tz. 2 der Interinstitutionellen Vereinbarung „Bessere Rechtsetzung“, ABI. EG Nr. C 321 vom 31. Dezember 2003, S. 1.

20 KOM (2002) 278 endgültig vom 5. Juni 2002.

21 KOM (2005) 97 endgültig vom 16. März 2005. 
verspricht. Dazu gehört zunächst ein Ausbau der Folgenabschätzung für vorgeschlagene Regelungen, die auch eine Quantifizierung der Verwaltungskosten umfassen soll22. Die Kommission bekundet ferner ihre Absicht, schwebende Rechtsetzungsvorschläge intensiver als bisher zu prüfen und ggf. auch zurückzuziehen. Schließlich kündigt sie Bemühungen zu einer Vereinfachung des bestehenden EU-Rechts an und verweist in diesem Zusammenhang auf ihre Mitteilung vom Februar 2003 zur „Aktualisierung und Vereinfachung des Acquis communautaire" 23.

So begrüßenswert diese Maßnahmen und Absichtserklärungen auch sein mögen - zu einer spürbaren Verbesserung der Gesetzgebungsqualität haben sie bislang nicht geführt. Sollte es dafür noch eines Beleges bedurft haben, so kann insoweit auf den Schlussantrag von Generalanwalt Geelhoed vom 5. April 2005 verwiesen werden, in dem mit deutlichen, ja fast schon sarkastischen Bemerkungen auf offenkundige Mängel in der Rechtsetzung der Gemeinschaft hingewiesen wird24. Es bedarf also fraglos weiterer Anstrengungen auf diesem Gebiet.

\section{b) Die Notwendigkeit eines unabhängigen Gesetzgebungs- und Beratungsdienstes}

Auf der Suche nach Wegen einer verbesserten Gesetzgebung der Gemeinschaft gilt es vor allem, die Schwierigkeiten europäischer Willensbildung und die daraus resultierende, besondere Kompromissprägung der gemeinschaftlichen Rechtsetzung zu bedenken.

22 Schon heute sind die von der Kommission vorgeschlagenen Rechtsakte Gegenstand einer integrierten Folgenabschätzung, zu denen jeweils im Vorfeld so genannte „Roadmaps“ veröffentlicht werden. Die Roadmaps für das Legislativ- und Arbeitsprogramm 2005 der Kommission sind einsehbar unter http://europa.eu.int/comm/secretariat_general/impact/ practice.htm.

23 Siehe dazu die Mitteilung der Kommission „Aktualisierung und Vereinfachung des Acquis communautaire" vom 11. Februar 2003, KOM (2003) 71 endgültig.

24 GA L. A. Geelhoed, Schlussanträge in den verb. Rechtssachen C-154/04 und C-155/04, noch nicht in amtl. Slg., insbesondere Rdnr. 72 und 88. Der Gerichtshof teilt die diesbezüglichen Bedenken seines Generalanwalts offenbar nicht, vgl. dazu das in der genannten Rechtsache ergangene Urteil vom 12. Juli 2005, noch nicht in amtl. Slg. 
Diese Besonderheiten der gemeinschaftlichen Rechtsetzung lassen das Fehlen einer formalisierten Rechtsförmlichkeitsprüfung ebenso deutlich wie negativ hervortreten. Wichtiger noch als ein entsprechendes formales Testat für die Rechtsetzungsvorschläge der Kommission, das ihrem Juristischen Dienst zu überantworten wäre, erscheint die Gewährleistung der Eindeutigkeit gewählter Begrifflichkeiten und die Beachtung der Rechtsförmlichkeit, der inneren Widerspruchsfreiheit und der systematischen Stimmigkeit von Gemeinschaftsrechtsakten im Laufe des weiteren Rechtsetzungsverfahrens. Insoweit rückt die Einrichtung eines unabhängigen Gesetzgebungs- und Beratungsdienstes auch auf europäischer Ebene in den Mittelpunkt der Überlegungen25. Entscheidend für eine praktisch wirksame Erfüllung dieser Aufgabenstellung in allen Stadien des Gesetzgebungsverfahrens ist ihre Einrichtung als weisungsfreie und verwaltungsorganisatorisch unabhängige Stelle26. Die Einrichtung eines unabhängigen Gesetzgebungs- und Beratungsdienstes hätte überdies den besonderen Charme, das bisher im Graubereich angesiedelte Wirken der Rechts- und Sprachsachverständigen27, das auch von einem solchen Gesetzgebungs- und Beratungsdienst wahrzunehmen wäre, auf eine institutionell zweifelsfreie Grundlage zu stellen.

25 Insoweit ist es zu begrüßen, dass der Juristische Dienst in Umsetzung der Interinstitutionellen Vereinbarung vom 22. Dezember 1998 über Leitlinien für die redaktionelle Qualität der gemeinschaftlichen Rechtsvorschriften (o. Fußn. 18), einen Leitfaden zur Abfassung von Rechtsvorschriften zur Verfügung gestellt hat. Siehe dazu Anhang I der Kommissions-Mitteilung KOM (2005) 97 endg. (o. Fußn. 21), S. 14.

26 Letztlich beruht diese Überlegung auf dem Grundsatz funktionsgerechter Organstruktur, siehe Thomas von Danwitz, Der Grundsatz funktionsgerechter Organstruktur - Verfassungsvorgaben für staatliche Entscheidungsstrukturen und ihre gerichtliche Kontrolle, Der Staat 35 (1996), S. $329 \mathrm{ff}$.

27 Dazu jüngst Ingolf Pernice, Einigungsmängel im EUMitentscheidungsverfahren - Zum Umgang mit Korrekturen durch die Rechts- und Sprachdienste der Institutionen, EuZW 2004, S. 743 (744 ff.); eingehend zu den sich aus der Mehrsprachigkeit ergebenden Problemen der Rechtsetzung Isabel Schübel-Pfister, Sprache und Gemeinschaftsrecht, 2004, insbesondere S. 92 ff. 


\section{c) Subsidiaritätskontrolle}

Ein zweites Element zur Verbesserung der gemeinschaftlichen Rechtsetzung lässt sich aus den Ansätzen gewinnen, die im Verfassungsvertrag für die Subsidiaritätskontrolle vorgesehen sind. Nach Art. 5 des Protokolls über die Anwendung der Grundsätze der Subsidiarität und der Verhältnismäßigkeit sollte jeder Entwurf eines Gesetzgebungsaktes einen Vermerk mit detaillierten Angaben enthalten, die eine Beurteilung ermöglichen, ob die Anforderungen von Subsidiarität und Verhältnismäßigkeit eingehalten werden. Neben Angaben zu den voraussichtlichen finanziellen Auswirkungen soll er auch zu den Auswirkungen der von den Mitgliedstaaten zu erlassenden Rechtsvorschriften und ggf. von regionalen Vorschriften Stellung nehmen und, soweit möglich, neben qualitativen auch auf quantitativen Kriterien beruhen. Auf diese Weise soll die Feststellung ermöglicht werden, ob ein bestimmtes Ziel besser auf der Unionsebene erreicht werden kann und die Belastungen sowie der von dem Gesetzgebungsakt veranlasste Verwaltungsaufwand dazu in einem angemessenen Verhältnis stehen.

Fraglos handelt es sich dabei um ein überaus ambitioniertes Anforderungsprofil, das wesentlich dazu beitragen kann, die Erforderlichkeit eines Gesetzgebungsaktes, aber auch die regelungstechnische Umsetzung der jeweiligen legislativen Zielsetzung einer analytischen Bewertung zu unterziehen. Bereits die Erarbeitung eines entsprechenden Vermerks von Seiten der zuständigen Kommissionsdienststellen dürfte das Problembewusstsein schärfen und dadurch zu einer qualitativen Verbesserung der Rechtsetzungsvorschläge beitragen. Allerdings müssten die Ausführungen ebenso detailliert wie aussagekräftig erfolgen und ganz auf die abstrakte Formelhaftigkeit verzichten, die auf den Vorblättern zu den Gesetzentwürfen der Bundesregierung ebenso wie in der bisherigen Kommissionspraxis vorherrschen und diese Darstellung zu einer fragwürdigen Formalie haben verkommen lassen28. Während solche Absichtserklärungen bisher allzu oft nur als placebo für Integrationskritiker gemeint waren, geht es spätestens nach den gescheiterten Referenden darum, diese Versprechen tatsächlich einzulösen. Ein Festhalten an dem bisherigen Kurs wäre fraglos ein echter Grund, sich um die Zukunft der europäischen Integ-

28 Zur Kritik an dieser Praxis etwa Schneider (o. Fußn. 4), § 5 Rn. 113. 
ration zu sorgen. Ebenso dringlich ist jedoch die Klärung der Anforderungen, die der Verhältnismäßigkeitsgrundsatz auf Gemeinschaftsebene tatsächlich stellt, und inwieweit diese der gerichtlichen Nachprüfung unterliegen29.

Gegenüber den unbestreitbaren Verbesserungen, die eine ernsthafte Ausführung dieser Vorgaben im Rahmen der Subsidiaritätsprüfung mit sich bringen würde, erscheint die in diesem Protokoll vorgesehene Verantwortungszuweisung an die Kommission und an die mitgliedstaatlichen Parlamente im Rahmen des Frühwarnsystems nach Art. 6 und 7, zumal wegen des vorausgesetzten Quorums von einem Drittel der Parlamente, als wenig gelungen. Auch insoweit erscheint es empfehlenswert, zu dem Vermerk der Kommissionsdienststellen das Votum eines unabhängigen Gremiums im Vorfeld der Beschlussfassung des Kommissionskollegiums einzuholen, das aus ehemaligen Richtern der Gemeinschaftsgerichte sowie der obersten mitgliedstaatlichen Gerichte und von Hochschullehrern zu bilden wäre. Sein Votum sollte als Grundlage für eine substantielle Diskussion der Vor- und Nachteile der jeweiligen Rechtsetzungsinitiative dienen und eine effektive gerichtliche Überprüfung erleichtern.

Eine fachliche Stellungnahme dieser Art würde es insbesondere ermöglichen, Rechtsetzungsinitiativen bereits im Vorfeld des förmlichen Gesetzgebungsverfahrens kritisch zu bewerten, die keinen nennenswerten integrativen Mehrwert für eine Verwirklichung des Binnenmarktes und die Ausführung der Gemeinschaftspolitiken erkennen lassen. Unsinnigerweise sind es in der Vergangenheit und Gegenwart nämlich vor allem solche Rechtsetzungsvorhaben gewesen, die keine zureichende Akzeptanz von Seiten der Mitgliedstaaten und ihren Bevölkerungen gefunden, aber erhebliche Kräfte gebunden haben. Der integrative Mehrwert von Tabakwerbeverboten30, von weitreichenden Verboten für nährwert- und ge-

29 Dazu bereits Thomas von Danwitz, Der Grundsatz der Verhältnismäßigkeit im Gemeinschaftsrecht, EWS 2003, S. 393 ff.; ferner Oliver Koch, Der Grundsatz der Verhältnismäßigkeit in der Rechtsprechung des Gerichtshofs der Europäischen Gemeinschaften, 2003; Katja Hauke, Verhältnismäßigkeit im europäischen Wirtschaftsverwaltungsrecht, 2004.

30 Richtlinie 89/622/EWG des Rates vom 13. November 1989 zur Angleichung der Rechts- und Verwaltungsvorschriften der Mitgliedstaaten über die Etikettierung von Tabakerzeugnissen sowie zum Verbot bestimmter 
sundheitsbezogene Angaben über Lebensmittel31 und von Regelungen über die Verpflichtung zur Einrichtung unternehmensexterner Fonds für die Stilllegung von Kernkraftwerken32 für die Verwirklichung des Binnenmarktes ist allenfalls gering; die Beurteilung des gesundheits-, des verbraucherschutz- und des energiepolitischen Mehrwertes dieser Rechtsetzungsakte sollte jedoch zuständigkeitsgemäß bei den Mitgliedstaaten verbleiben33. Eine Kon-

Tabake zum oralen Gebrauch, geändert durch Richtlinie 92/41/EWG des Rates (sowie Richtlinie 90/239/EWG des Rates vom 17. Mai 1990 zur Angleichung der Rechts- und Verwaltungsvorschriften der Mitgliedstaaten über den höchstzulässigen Teergehalt von Zigaretten), aufgehoben durch Art. 15 Richtlinie 2001/37/EG des Europäischen Parlaments und des Rates vom 5. Juni 2001 zur Angleichung der Rechts- und Verwaltungsvorschriften der Mitgliedstaaten über die Herstellung, die Aufmachung und den Verkauf von Tabakerzeugnissen. Richtlinie 2003/33/EG des Europäischen Parlaments und des Rates vom 26. Mai 2003 zur Angleichung der Rechts- und Verwaltungsvorschriften der Mitgliedstaaten über Werbung und Sponsoring zugunsten von Tabakerzeugnissen, $\mathrm{ABI}$. Nr. L 152 vom 20. 6. 2003 S. 16. Die Richtlinie 98/43/EG des Europäischen Parlaments und des Rates vom 6. Juli 1998 zur Angleichung der Rechts- und Verwaltungsvorschriften der Mitgliedstaaten über Werbung und Sponsoring zugunsten von Tabakerzeugnissen, ABI. Nr. L 213 vom 30. 7. 1998 S. 9, wurde vom Gerichtshof für nichtig erklärt, Rs. C-376/98. Vgl. auch die Entscheidung der Kommission vom 5. September 2003 über die Verwendung von Farbfotografien oder anderen Abbildungen als gesundheitsbezogene Warnhinweise auf Verpackungen von Tabakerzeugnissen, ABI. Nr. L 226 vom 10. 09. 2003 S. 24.

31 Vorschlag der Kommission für eine Verordnung über nährwert- und gesundheitsbezogene Angaben über Lebensmittel vom 16. 7. 2003, KOM (2003) 424 endg.

32 Vorschlag der Kommission für eine Richtlinie (Euratom) des Rates zur Festlegung grundlegender Verpflichtungen und allgemeiner Grundsätze im Bereich der Sicherheit kerntechnischer Anlagen vom 30. Januar 2003, KOM (2003) 32 endgültig.

33 Dass die Gemeinschaft auf den Gebieten des Gesundheits- und des Verbraucherschutzes nur über eingeschränkte Kompetenzen verfügt, folgt bereits aus Art. $152 \mathrm{f}$. EG und ist allgemein anerkannt. Siehe etwa Brigitta Lurger, in: Streinz (Hrsg.), EUV/EGV, Kommentar, 2003, Art. 153 Rn. 2. Das gilt erst recht für den Energiebereich. Dazu Jens-Peter Schneider, in: Schneider/Theobald (Hrsg.), Handbuch zum Recht der Energiewirtschaft, 2003, § 2 Rn. 6 ff. Vgl. für den Bereich der Kultur eingehend Thomas von Danwitz, Die Kultur in der Verfassungsordnung der Europäischen Union, NJW 2005, S. 529 ff. 
zentration der Gemeinschaft auf ihre Kernkompetenzen wird fraglos zu einer verbesserten Akzeptanz des Gemeinschaftshandelns beitragen. Dies gilt namentlich nach den misslungenen Referenden in Frankreich und den Niederlanden. Vor diesem Hintergrund ist die Ankündigung der Kommission zu begrüßen, im Laufe des Jahres 2005 eine Gruppe von hochrangigen nationalen Rechtsetzungssachverständigen einsetzen zu wollen, die den Auftrag erhalten soll, die Kommission in allgemeinen Fragen der Rechtsetzung zu beraten34.

\section{d) Rücknahme der Regelungsdichte gemeinschaftlicher Richtli- nien}

Ein dritter Weg zur Verbesserung der europäischen Rechtssetzung besteht in der Rückführung der Regelungsdichte gemeinschaftlicher Richtlinien. Dass sich die Richtlinien entgegen ihrer primärrechtlichen Funktionsbeschreibung seit geraumer Zeit nicht mehr darauf beschränken, als bloßes Instrument zur gemeinschaftlichen Festlegung verbindlicher Zielvorgaben zu fungieren, das den Mitgliedstaaten die Wahl der zu ihrer Erreichung dienlichen Form und der Mittel belässt, ist schon ein gemeinschaftsrechtlicher Allgemeinplatz35. Richtlinien haben sich weitgehend zu vollständigen Normsetzungsprogrammen für den geregelten Sachbereich entwickelt, die alle wesentlichen Aspekte einer innerstaatlichen Regelung behandeln36. In dieser Zunahme der normativen Regelungsdichte von Richtlinien liegt die eigentliche Erklärung dafür, warum die Rechtsinstitute der unmittelbaren Wirkung von Richtlinien und der richtlinienkonformen Auslegung des innerstaatlichen Rechts sich zu Phänomenen mit einer solchen Breitenwirkung entwickelt haben. Durch das Zusammenwirken dieser Faktoren können die Richtlinien in Ermangelung einer ordnungsgemäßen Umsetzung auch innerstaatlich Wirkungen entfalten, die sich als effektives Ge-

34 Siehe dazu die Kommissions-Mitteilung KOM (2005) 97 endgültig (o. Fußn. 21), S. 11.

35 Statt aller etwa Matthias Ruffert, in: Callies/Ruffert (Hrsg.), EUV/EGV, Kommentar, 2. Auflage 2002, Art. 249 EGV Rn. 45; Werner Schroeder, in: Streinz (Hrsg.), EUV/EGV, Kommentar, 2003, Art. 249 EGV Rn. 69.

36 Treffend Peter M. Huber, Recht der Europäischen Integration, 2. Auflage 2002, \& 8 Rn. 97: Nationale Parlamente in der Rolle von Notaren. 
gengewicht zu den vielfältigen Defiziten der mitgliedstaatlichen Richtlinienumsetzung erweisen.

Angesichts der beständigen Säumnis der Mitgliedstaaten bei der Umsetzung von Richtlinien, für die die Mitgliedstaaten - unabhängig von der gemeinschaftsrechtlichen Unbeachtlichkeit solcher Erklärungen - zu selten nachvollziehbare Gründe anführen können37, wird man der hohen Regelungsdichte der Gemeinschaftsrichtlinien aus europäischer Perspektive oft genug sogar viel Verständnis entgegen bringen müssen. Insbesondere hat diese Entwicklung bisher nicht $\mathrm{zu}$ einem Rechtsformenmissbrauch des Rechtssetzungsinstruments der Richtlinie geführt, denn selbst Richtlinien mit einem vollständigen Normsetzungsprogramm belassen den Mitgliedstaaten mehr oder weniger bedeutsame Ausführungs- und Umsetzungsspielräume und bedürfen zudem einer systemgerechten Einpassung in die sie umgebenden Vorschriften des mitgliedstaatlichen Rechts38. Daher bleibt die en-blocUmsetzung von Richtlinien in der italienischen Staatspraxis durch ein jährliches „legge communiaria“, die auf die berühmte legge La Pergola aus dem Jahre 1989 zurückgeht, gemeinschaftsrechtlich unzureichend. Jenseits der fehlenden Einpassung der Richtlinien in die italienische Rechtsordnung enthalten derartige Artikelgesetze auch der Sache nach nur Ermächtigungen an die Regierung, die erforderlichen Umsetzungsmaßnahmen zu treffen39.

Trotz der nachvollziehbaren Gründe, die zu der hohen Regelungsdichte von Gemeinschaftsrichtlinien geführt haben, bleibt festzuhalten, dass auf diese Weise die mitgliedstaatliche Aufgabe der systemkonformen und möglichst nahtlosen Einpassung der gemeinschaftlichen Angleichungsanordnung in die Struktur und Systematik des innerstaatlichen Rechts mit einer Vielzahl von Vorgaben befrachtet wird, die der mitgliedstaatlichen Angleichungsaufgabe erhebliche Schwierigkeiten bereitet 40 . Die subsidiaritätsrecht-

37 Dazu mit Nachweisen schon Thomas von Danwitz, Die Nichtumsetzung von EG-Recht durch die Legislative, NWVBL 1997, S. 7(8).

38 Siehe von Danwitz (o. Fußn. 15), Rn. 88 f.

39 Siehe Adinolfi, The judicial application of community law in Italy (19811997), CMLRev. 35 (1998), S. 1313 (1314).

40 Dazu Thomas von Danwitz, Europarechtliche Determination der umweltrechtlichen Instrumente im nationalen Recht, in: Rückzug des Ordnungsrechtes im Umweltschutz, Tagungsband des 14. Trierer Kolloquiums 
liche Forderung nach Öffnungs-, Kompensations- und Äquivalenzklauseln, die bereits im Subsidiaritätsprotokoll des Amsterdamer Vertrages erhoben wurde41, ist bisher allerdings ohne praktische Konsequenzen geblieben. Die durch die beständige Säumnis der umsetzungsverpflichteten Mitgliedstaaten ausgelöste Regelungsdichte der Gemeinschaftsrichtlinien führt zu erheblichen Umsetzungsschwierigkeiten, namentlich im Hinblick auf ihre systemkonforme Einpassung in das mitgliedstaatliche Recht, wodurch es wiederum zu qualitativen Umsetzungsdefiziten und Friktionen mit dem innerstaatlichen Recht kommt, die der praktischen Wirksamkeit des Gemeinschaftsrechts oftmals mehr als nur abträglich sind.

Diese Entwicklung beschreibt einen Teufelskreis, der nur durchbrochen werden kann, wenn die Mitgliedstaaten ihre strukturellen Schwierigkeiten mit der Erfüllung ihrer Umsetzungsverpflichtung entschlossen angehen und die Gemeinschaft das Vertrauen in die Erfüllung der Umsetzungsverpflichtung durch die Einräumung entsprechender Freiräume zu honorieren bereit ist. Jedenfalls ist die Zeit gekommen, von überzogenen Einheitlichkeitsvorstellungen Abschied zu nehmen und Rechtsangleichung nicht als Selbstzweck zu begreifen, die der Schaffung von Einheitsrecht dienen soll, sondern sich auf ihre vertragliche Aufgabenstellung zu besinnen, tatsächliche Hindernisse für die Verwirklichung der Grundfreiheiten und spürbare Wettbewerbsverfälschungen zu beseitigen 42 . Auch bei so viel Selbstbeschränkung würde der gemeinschaftlichen Rechtsangleichung in den nächsten 50 Jahren die Arbeit nicht ausgehen.

zum Umwelt- und Technikrecht, 06. bis 08.09.1998, 1999, S. 53 (72 ff.); Rüdiger Breuer, Der Entwurf der EG-Wasserrahmenrichtlinie, NVwZ 1998, S. $1001 \mathrm{ff}$.

41 So Ziffer 7 a. E. des Subsidiaritätsprotokolls Nr. 21 vom Amsterdam. Dazu von Danwitz (o. Fußn. 40), S. 81 f.

42 Siehe schon von Danwitz (o. Fußn. 15), Rn. 100 ff., 104; aus der Rechtsprechung des Gerichtshofs insbesondere EuGH, Slg. 2000, I-8419 (8530; Rn. 107 ff.) - Tabakwerbeverbot; Urteil vom 14. Dezember 2004 in der Rs. C-434/02, Arnold André GmbH, Rn. 29 ff. 


\section{Die Ebene mitgliedstaatlicher Mitwirkung an der Gemein- schaftsrechtsetzung}

Wenden wir uns nun den Möglichkeiten zu, im Rahmen der mitgliedstaatlichen Mitwirkung an der Gemeinschaftsrechtsetzung zu einer Verbesserung der redaktionellen und legistischen Qualität gemeinschaftlicher Rechtsakte beizutragen.

\section{a) Die Mitwirkung von Bundestag und Bundesrat nach Art. 23 GG}

In Deutschland kreist die Diskussion um die Mitwirkung an der gemeinschaftlichen Rechtsetzung im Rat naturgemäß um die Komplexität der Beteiligung von Bundestag und Bundesrat nach Art. 23 Abs. 3 bis 7 GG und den dazu ergangenen Ausführungsgesetzen43. Es liegt auf der Hand, dass eine so weitgehende Beteiligung des Bundestages und vor allem auch des Bundesrates zu Lasten der Effizienz der deutschen Mitwirkung an der Gemeinschaftsrechtsetzung gehen kann. Im Ausgangspunkt ist indes zu bedenken, wie wesentlich die nach Art. 23 GG stattfindende Betei-

43 Dazu etwa Udo Di Fabio, Der neue Art. 23 des Grundgesetzes, Der Staat 32 (1993), S. 191 ff.; Kirstin Schmalenbach, Der neue Europaartikel 23 des Grundgesetzes im Lichte der Arbeit der gemeinsamen Verfassungskommission, 1996; Ralf Müller-Terpitz, Die Beteiligung des Bundesrates am Willensbildungsprozess der Europäischen Union, 1999. Das Gesetz über die Zusammenarbeit von Bundesregierung und Deutschem Bundestag in Angelegenheiten der Europäischen Union vom 12. März 1993 (BGBI. I 311) sowie das Gesetz über die Zusammenarbeit von Bund und Ländern in Angelegenheiten der Europäischen Union vom gleichen Tage (BGBI. I 313, 1780) sind im Zuge der Ratifikation des Verfassungsvertrages geändert und durch ein Gesetz über die Ausübung der Rechte des Bundestages und des Bundesrates aus dem Vertrag vom 29. Oktober 2004 über eine Verfassung für Europa ergänzt worden. Siehe dazu die Art. 1 und 2 des Gesetzes über die Ausweitung der Stärkung der Rechte des Bundestages und des Bundesrates in Angelegenheiten der Europäischen Union, dem der Bundestag am 12. Mai 2005 (BR-Drs. 340/05) und der Bundesrat am 27. Mai 2005 (BR-Drs. 340/05 [Beschluss]) zugestimmt haben. Das Gesetz wird im Wesentlichen an dem Tag in Kraft treten, an dem der Vertrag über eine Verfassung für Europa nach seinem Artikel IV-447 Abs. 2 für die Bundesrepublik in Kraft tritt (Art. 3 des Gesetzes über die Ausweitung der Stärkung der Rechte des Bundestages und des Bundesrates). 
ligung der gesetzgebenden Körperschaften für die notwendige demokratische Legitimation der Gemeinschaftsrechtsetzung über die deutsche Mitwirkung im Rat gerade aus verfassungsrechtlicher Perspektive ist 44 . Dennoch ist es im Rahmen der gemeinschaftlichen Rechtsetzung praktisch von Bedeutung, gegenüber der Kommission bereits vor Verabschiedung eines Rechtsetzungsvorschlages Position beziehen zu können und namentlich in den Arbeitsgruppen des Rates frühzeitig eine innerstaatlich abgestimmte Position zu vertreten, die auch Alternativvorschläge und regelungstechnische Einzelfragen erfasst.

Für die Mehrheits- und Kompromissbildung mit den Vertretern der anderen Mitgliedstaaten im Rat und für ein koordiniertes Auftreten gegenüber Kommission und Parlament ist es fraglos von elementarer Bedeutung, die innerstaatliche Meinungsbildung möglichst frühzeitig abgeschlossen zu haben. Dennoch ist der oftmals vermittelte Eindruck45 unzutreffend, die beachtliche Mitwirkung des Bundesrates bilde das alleinige oder vordringliche Hindernis für eine effiziente Mitwirkung der Vertreter Deutschlands im Rat. Vielmehr sind auch die Beispiele einer koalitionspolitischen Neutralisierung der deutschen Mitwirkung im Rat zu beklagen, die in jüngerer Zeit namentlich im Energie- und Umweltbereich aufgetreten sind. Das Streben nach wirkungsvoller Mitwirkung an der Gemeinschaftsrechtsetzung stellt daher auch die Koalitionsdisziplin vor besondere Anforderungen. Insgesamt scheint mir weniger eine Flurbereinigung der Mitwirkung von Bundestag und Bundesrat angezeigt als vielmehr eine Straffung der vorgesehenen Beteiligungsfristen und die Intensivierung der Koordinierung zwischen Bund und Ländern.

44 Siehe BVerfGE 89, 155 (182 ff.) - Maastricht; 97, 350 (368 ff.) - Währungsunion.

45 Dazu zuletzt die Darstellung bei Rudolf Hrbek, Der deutsche Bundesstaat in der EU - Die Mitwirkung der deutschen Länder in EUAngelegenheiten als Gegenstand der Förderalismus-Reform, in: FS Zuleeg, 2005, S. 256 (261 ff.). 


\section{b) Zur redaktionellen und legistischen Qualität der Gemein- schaftsrechtsetzung}

Ein weiterer, für die Qualität der Gemeinschaftsrechtsetzung maßgeblicher Punkt betrifft die Bereitschaft der im Rat vertretenen Mitgliedstaaten, aktiv auf die Gemeinschaftsrechtsetzung einzuwirken, um in redaktioneller und rechtstechnischer Hinsicht den Erlass von gemeinschaftsrechtlichen Vorschriften zu erreichen, die gegenüber den entsprechenden Vorschriften des nationalen Rechts kompatibel ausgestaltet sind. Angesichts von schon bald annähernd 30 Mitgliedstaaten kann es naturgemäß nicht darum gehen, das Regelungsmodell eines bestimmten Mitgliedstaates „in Reinkultur" durchzusetzen. Der Respekt vor den verschiedenen rechtsstaatlichen Traditionen in Europa und ihren mitgliedstaatlichen Ausprägungen bedingt eine entsprechende Zurückhaltung für einzelne Mitgliedstaaten ebenso wie für die Kommission. Die gemeinschaftsrechtliche Übernahme eines bestimmten mitgliedstaatlichen Modells kann nämlich für alle anderen einen erheblichen regelungstechnischen Umstellungsaufwand bedeuten, der die Qualität der Umsetzungsgesetzgebung wesentlich beeinträchtigen und im Zweifel sogar die praktische Wirksamkeit dieses Umsetzungsrechts in Mitleidenschaft ziehen wird.

Es kann also nicht darum gehen, die Vorzüge der eigenen Rechtsstrukturen in Europa durchzusetzen. Vielmehr muss die rechtstrukturelle und redaktionelle Abfassung der Vorschriften des Gemeinschaftsrechts in einer Weise gewährleistet werden, die ihrer Inkorporation in das mitgliedstaatliche Recht jedenfalls keine zusätzlichen Hindernisse bereitet. Es liegt auf der Hand, dass die Mitgliedstaaten in dieser Hinsicht weitgehend selbst ihres Glückes Schmied sind und man von innen erwarten muss, dass sie etwaige Schwierigkeiten artikulieren und entsprechende Vorschläge vorlegen, um diesen zu begegnen.

Voraussetzung ist allerdings, dass bereits die Verhandlungen in den Arbeitsgruppen des Rates mit der entsprechenden Umsetzungsorientierung und im Bewusstsein um die Bedeutung geführt werden, die einer umsetzungsgeeigneten strukturellen und redaktionellen Abfassung des sekundären Gemeinschaftsrechts zukommt. In dieser Hinsicht habe ich bisher kein ausgeprägtes Problembewusstsein bei deutschen Bundesbeamten verschiedener Fachressorts feststellen können. Dies gilt auch für das Umweltres- 
sort, obwohl es diese Lektion als gebranntes Kind doch eigentlich gelernt haben sollte. Mitunter versuchen Amtsträger auch heute noch den selbstverständlich unzutreffenden Eindruck zu vermitteln, ausgerechnet die Regelungsstruktur und der redaktionelle Duktus der Kommissionsvorschläge seien gleichsam sakrosankt. Ein instruktives Beispiel für die Schwierigkeiten, die durch die strikte Verfolgung einer bestimmten Regelungskonzeption im Gemeinschaftsrecht ausgelöst werden, ist der verfahrensrechtliche Grundansatz der Kommissionsvorschläge für Rechtsetzungsvorhaben im Umweltbereich, die weitgehend den Regelungsstrukturen des britischen Rechts folgen46 und in einem klaren konzeptionellen Gegensatz zu den materiell-rechtlichen Regelungsstrukturen des deutschen Umweltrechtes stehen47. Normativ wird dieser Konzeptionsunterschied durch den Gegensatz zwischen finalstrukturierten Vorschriften des gemeinschaftlichen Umweltrechts und konditional aufgebauten Bestimmungen des deutschen Umweltrechts zum Ausdruck gebracht. Dieser konzeptionelle Unterschied und seine normstrukturelle Umsetzung haben der Rechtsetzung im Umweltrecht seit dem Beginn der 90er Jahre vielfältige, mitunter kaum auflösbar erscheinende Schwierigkeiten bereitet 48 und zu mehrfachen Verurteilungen Deutschlands wegen Vertragsverletzungen durch den Europäischen Gerichtshof geführt49.

Dieser hinlänglich bekannte Befund ist umso erstaunlicher, als es in keinem dieser Fälle um eine inhaltliche Verfehlung der gemeinschaftsrechtlichen Umweltanforderungen, sondern - ich überzeichne - um die regelungstechnische Ausgestaltung des harmo-

46 Zum wachsenden Einfluss des anglo-skandinavischen Modells auf das europäische Umweltrecht etwa Michael Kloepfer, Die europäische Herausforderung - Spannungslagen zwischen deutschem und europäischem Umweltrecht, NVwZ 2002, S. 645 (647).

47 Siehe Rüdiger Breuer, Umsetzung von EG-Richtlinien im neuen Energiewirtschaftsrecht, NVwZ 2004, S. 520 (523 ff.).

48 Phänotypisch erscheint insoweit in der Rückschau der Prozess zur Umsetzung der UVP-Richtlinie. Dazu etwa Rüdiger Breuer, Entwicklungen des Europäischen Umweltrechts, 1993, S. $51 \mathrm{ff}$.

49 Siehe etwa EuGH, Slg. 1991, 825 ff. - Grundwasserrichtlinie; Slg. 1991, 2567 ff. - Schwefeldioxid und Schwebestaub; Slg. 1991, 2607 ff. - Blei; Slg. 1991, 4983 ff. - Trinkwasser; Slg. 1998, I-6135 - UVPG; Slg. 1999, I-5153 ff. - UIG. Vgl. auch die Darstellung bei Kloepfer (o. Fußn. 46), NVwZ 2002, S. 645 (647 f.). 
nisierten Umweltrechts gegangen ist. Nun mag die mitunter geäußerte Auffassung zutreffend sein, dass die materielle Umweltrechtskonzeption deutscher Tradition in Europa nicht mehrheitsfähig und der verfahrensrechtliche Grundansatz auf Gemeinschaftsebene gleichsam unvermeidlich gewesen sei50. Dann hätte es aus deutscher Sicht aber mehr als nur nahe gelegen, in den Verhandlungen des Rates auf die Aufnahme einer Bestimmung über die funktionale Äquivalenz dieser unterschiedlich strukturierten Regelungsansätze hinzuwirken oder Regelungs- und Formulierungsvorschläge mit dem Ziel einer rechtstechnisch weitgehenden Kompatibilität der zu erlassenden Gemeinschaftsregelungen mit den bestehenden Strukturen des deutschen Umweltrechts in die Verhandlungen einzubringen. Eine derartige Verhandlungsstrategie, die wegen ihrer grundsätzlichen Unterstützung für die Regelungsvorschläge der Kommission prinzipiell nicht aussichtslos sein dürfte, ist bisher offenbar aber nicht verfolgt worden, obwohl sie letztlich nur darauf abgezielt hätte, Reibungsverlusten sowie Unstimmigkeiten im Rahmen der Umsetzung präventiv zu begegnen und auf diese Weise zur praktischen Wirksamkeit der Rechtsangleichung beizutragen.

Ebenso wenig ist im Rahmen der Umsetzung sekundärrechtlicher Vorgaben der weiteren Grundfrage besondere Aufmerksamkeit geschenkt worden, wie im Zuge einer verpflichtenden Übernahme der verfahrensrechtlichen Grundkonzeption des gemeinschaftlichen Umweltrechts mit den materiell-rechtlichen Anforderungen des tradierten deutschen Umweltrechts zu verfahren sei. Obwohl sie sich geradezu aufdrängt, ist die strategische Alternative einer ggf. modifizierten - Fortführung der überkommenen Strukturen des deutschen Umweltrechts oder ihre Aufgabe unter Vollziehung eines grundlegenden Paradigmenwechsels zugunsten des gemeinschaftsrechtlichen Grundansatzes einer weitgehend verfahrensrechtlichen Konzeption zu keiner Zeit eingehend diskutiert worden51. Vielmehr ist aus der gemeinschaftsrechtlichen Vorgabe ei-

50 Siehe dazu den nüchternen Befund bei Rüdiger Breuer, Zunehmende Vielgestaltigkeit der Instrumente im deutschen und europäischen Umweltrecht - Probleme der Stimmigkeit und des Zusammenwirkens, NVwZ 1997, S. 833 (837 f.).

51 Vgl. aber etwa Breuer (o. Fußn. 50), NVwZ 1997, S. 833 (838 ff.); siehe ferner Martin Gellermann, Beeinflussung des bundesdeutschen Rechts 
ner verfahrensrechtlichen Ausrichtung des angeglichenen Umweltrechts die Notwendigkeit abgeleitet worden, diese Vorgaben auf den ungeschmälerten Bestand des deutschen Umweltrechts überkommener Prägung „aufzusatteln“. Jedoch sind die Konsequenzen dieser Kumulierung regulatorischer Vorgaben im Hinblick auf die Komplexität und Regelungsdichte der normativen Vorgaben, aber auch für die Wettbewerbsbedingungen der deutschen Wirtschaft im Binnenmarkt, nicht gebührend berücksichtigt und bewertet worden.

\section{Die innerstaatliche Umsetzung gemeinschaftlicher Vorgaben}

Die innerstaatliche Umsetzung des gemeinschaftlichen Richtlinienrechts bildet seit geraumer Zeit ein reichlich trübes Kapitel der europäischen Integration. Die verspätete oder ungenügende Umsetzung erscheint in manchen Mitgliedstaaten mehr als Regel denn als Ausnahme. Auch für Deutschland ist die Erfüllung seiner Umsetzungsverpflichtungen keineswegs ein Ruhmesblatt52. Neben den bekannten Fällen offener Obstruktion53 und schlichter Nachlässigkeit der Mitgliedstaaten haben sich in der Zwischenzeit vielfach auch objektive Umsetzungsschwierigkeiten herauskristallisiert, die gemeinschaftsrechtlich unentschuldbar bleiben, aber gleichwohl sachlich bedingt sind. Benachteiligt sind fraglos Mitgliedstaaten, die eine komplexe innerstaatliche Zuständigkeitsordnung aufweisen. Gleiches gilt für mitgliedstaatliche Verfassungs-

durch Richtlinien der EG. Dargestellt an Beispielen des europäischen Umweltrechts, 1994; Christoph Demmke, Die Implementation von EGUmweltpolitik in den Mitgliedstaaten, 1994; Klaus Hansmann, Schwierigkeiten bei der Umsetzung und Durchführung des europäischen Umweltrechts, NVwZ 1995, S. 320 ff.; Rudolf Steinberg, Probleme der Europäisierung des deutschen Umweltrechts, AöR 120 (1995), S. 549 ff.

52 Dazu bereits von Danwitz (o. Fußn. 37), NWVBL 1997, S. 7 ff. Alljährliche Kommissionsberichte über die Kontrolle der Einhaltung des Gemeinschaftsrechts werden seit 1983 erstellt. Siehe aus den letzten Jahren den 19. Jahresbericht über die Kontrolle der Anwendung des Gemeinschaftsrechts, ABI. EG Nr. C 214 vom 10. 9. 2002, S. 21 (KOM (2002) 324 endg. vom 28. 6. 2002), den 20. Jahresbericht (KOM (2003) 669 endg. vom 21. 11. 2003) und den 21. Jahresbericht (KOM (2004) 839 endg. vom 30. 12. 2004).

53 Hier sei an die berühmte guerre de mouton erinnert, siehe $E u G H$, Slg. 1979, 2729 ff. - Schaffleisch. 
ordnungen, in denen die Exekutive über keine autonome Verordnungsgewalt verfügt. Die Umsetzung hängt in diesen Mitgliedstaaten besonders stark von der parlamentarischen Rechtsetzung ab. In kleineren Mitgliedstaaten ist schließlich schon die Umsetzung des ambitionierten Binnenmarktprogramms auf objektive Grenzen ihrer Leistungsfähigkeit gestoßen54. Als zukünftig wichtigster Grund für Defizite und Verzögerungen von Umsetzungsmaßnahmen ist indes die besondere Komplexität der innerstaatlichen Regelungen in den betroffenen Rechtsbereichen zu nennen55.

\section{a) Das Problem der mitgliedstaatlichen Ergänzung gemeinschaft- licher Regelungen}

Vor dem Hintergrund der besonderen Komplexität vieler Rechtsbereiche ist das sog. „Aufsatteln“ der deutschen Gesetzgebungspraxis auf die durch das Richtlinienrecht der Gemeinschaft ausgelöste Umsetzungsverpflichtung zu einem wirklichen Problemfall der Rechtsetzung im europäischen Mehrebenensystem geworden. Diese Neigung des deutschen Bundesgesetzgebers ist dennoch weit verbreitet. So ist das Antidiskriminierungsgesetz bereits zum Politikum geworden56. Auch die Novellierung des Energiewirtschaftsrechts geht in wesentlicher Hinsicht über die Anforderungen des ihm zugrunde liegenden Richtlinienpaketes der Gemeinschaft hinaus57. Besonders illustrativ ist in dieser Hinsicht der nun vorliegende Gesetzentwurf für ein Umwelt-Rechtsbehelfsgesetz58.

54 Siehe für Luxemburg den 11. Jahresbericht der Kommission, ABI. EG Nr. C 154 vom 6. 6. 1994, S. 44.

55 Für das europäische Umweltrecht namentlich Breuer (o. Fußn. 48), S. $78 \mathrm{ff}$. und für das Steuerrecht 12. Jahresbericht der Kommission, ABI. EG Nr. C 254 vom 29. 9. 1995, S. 8.

56 Dazu zuletzt etwa Christian Armbrüster, Antidiskriminierungsgesetz - ein neuer Anlauf, ZRP 2005, S. 41 ff. mit zahlreichen w. Nachw.

57 So schreibt Art. 23 Abs. 2 lit a) der Richtlinie 2003/54/EG des Europäischen Parlaments und des Rates vom 26. Juni 2003 über gemeinsame Vorschriften für den Elektrizitätsbinnenmarkt und zur Aufhebung der Richtlinie 96/92/EG, ABI. EU Nr. L 176 vom 15. Juli 2003, S. 37 ff. im Hinblick auf die Zugangsentgelte lediglich eine Methodenregulierung zwingend vor. Im nationalen Umsetzungsprozess haben sich dagegen zuletzt die Befürworter einer deutlich verschärften Regulierung durchgesetzt, in der etwa auch die Einzelregulierung der Zugangsentgelte in Gestalt einer Entgeltgenehmigung vorgesehen ist. Siehe dazu den $\S 23 a$ 
Aus gemeinschaftsrechtlicher Perspektive ist insoweit daran zu erinnern, dass die Rechtsangleichung regelmäßig in Formen der optionalen, der fakultativen oder der Mindestharmonisierung stattfindet59, die einer weitergehenden Regelung von Seiten der Mitgliedstaaten prinzipiell nicht entgegen steht60. Dennoch ist zu bedenken, dass vor allem ein unter den Mitgliedstaaten nicht koordiniertes „Aufsatteln“ einzelner Länder nach eigenständigen rechtspolitischen Präferenzen zugleich eine Entfernung von dem konzeptionellen Leitmotiv der Beseitigung wirtschafts- und wettbewerbsrelevanter Rechtsunterschiede durch die Schaffung von einheitlichen rechtlichen Rahmenbedingungen bedeutet61. Ein unkoordiniertes „Aufsatteln“ einzelner Mitgliedstaaten kann sogar neue Wettbewerbsverfälschungen bewirken und auf diese Weise die integrative Wirkung einer Rechtsangleichungsmaßnahme beeinträchtigen oder unter Umständen in Frage stellen. Hinzu kommen weitere

EnWG, der erst im Vermittlungsverfahren Eingang in Art. 1 des Zweiten Gesetzes zur Neuregelung des Energiewirtschaftsrechts gefunden hat. Siehe dazu die Beschlussempfehlung des Vermittlungsausschusses vom 15. Juni 2005 (BT-Drs. 15/5736 (neu)), der der Bundestag am 16. Juni 2005 zugestimmt hat (BR-Drs. 498/05). Zu einer eingehenden Analyse der gemeinschaftsrechtlich zwingend vorgeschriebenen Regulierungsstandards statt vieler Ulrich Büdenbender, Die Ausgestaltung des Regulierungskonzepts für die Elektrizitäts- und Gaswirtschaft, RdE 2004, S. $284 \mathrm{ff}$.

58 Entwurf eines Gesetzes über ergänzende Vorschriften zu Rechtsbehelfen in Umweltangelegenheiten nach der EG-Richtlinie 2003/35/EG (Umweltrechtsbehelfsgesetz) vom 21. Februar 2005. Zugänglich über die Homepage des BMU http://www.bmu.de/files/buergerbeteiligungs rechte/downloads/application/pdf/umweltrechtsbehelfsgesetz.pdf.

59 Siehe von Danwitz (o. Fußn. 15), Rn. 84; Rudolf Streinz, Mindestharmonisierung im Binnenmarkt, in: Everling/Roth (Hrsg.), Mindestharmonisierung im Europäischen Binnenmarkt, S. 9 (18 f.); Wolfgang Kahl, in: Callies/Ruffert (Hrsg.), EUV/EGV, Kommentar, 2. Auflage 2002, Art. 94 EGV Rn. 4; Stefan Leible, in: Streinz (Hrsg.), EUV/EGV, Kommentar, 2003, Art. 95 EGV Rn. 39 ff.

60 Kahl (o. Fußn. 59), Art. 94 EGV Rn. 4; Leible (o. Fußn. 59), Art. 95 EGV Rn. 42.

61 Das „Aufsatteln“ bzw. die „Übererfüllung“, d. h. die Einführung von materiellen Anforderungen und Verfahren, die sich nicht automatisch aus der Richtlinie ergeben, ist auch auf Gemeinschaftsebene als Problem erkannt. Siehe dazu nur die Kommissions-Mitteilung KOM (2005) 97 endg. (o. Fußn. 21), S. 10. 
Schwierigkeiten, die mit einer Verzögerung der Umsetzung, einer erheblichen Steigerung der Regelungsdichte und der normativen Komplexität einhergehen. Die Anreicherung der Umsetzungsrechtsakte durch originär innerstaatliche Regelungsanliegen kann sogar zu einer weitreichenden Wesensveränderung führen, die in extremis den Grund für das Verdikt der Gemeinschaftsrechtwidrigkeit einer Umsetzungsmaßnahme bilden kann. Diesen Befund diskutieren wir zur Zeit für das neue TKG aus dem Jahre 200462.

\section{b) Vortäuschung von bestimmten Umsetzungsverpflichtungen}

Ungleich problematischer sind aber die Fälle, in denen die Regierung einen Gesetzentwurf unter dem Vorwand in das Gesetzgebungsverfahren einbringt, von Gemeinschaftsrechts wegen zu genau dieser Umsetzungsmaßnahme verpflichtet zu sein. Diese Erklärung bedeutet, dass Bundestag und Bundesrat insoweit über keinerlei inhaltlichen Umsetzungs- und Gestaltungsspielraum verfügen. Die mündliche Verhandlung des BVerfG zum sog. europäischen Haftbefehl hat in das öffentliche Bewusstsein gebracht63, dass diese Praxis der Vortäuschung einer gemeinschaftsrechtlichen Bindung offenbar nicht außergewöhnlich ist. Das UmweltRechtsbehelfsgesetz bildet einen weiteren aktuellen, wenngleich unspektakulären Fall einer derartigen Instrumentalisierung der ge-

62 Umstritten ist insbesondere, ob der nationale Gesetzgeber berechtigt war, die der Regulierungsbehörde gemeinschaftsrechtlich zugewiesenen Freiräume normativ auszufüllen. Dazu etwa Ulrich Ellinghaus, TKGNovelle und Europarecht: Probleme mit der Flexibilisierung, CR 2004, S. 23 ff.; Robert Klotz, Der Referentenentwurf zum TKG im Licht der europarechtlichen Vorgaben, MMR 2003, S. 495 ff.; Christian Koenig/Sascha Loetz/Andreas Neumann, Sektorspezifische Regulierung im neuen Telekommunikationsrecht - Umsetzungsspielräume, verfassungsrechtliche Vorgaben und Verfahrensgestaltung, Beilage 2 zu K \& R 2003, S. 1 ff.

63 Während dieser Verhandlung hatten mehrere Abgeordnete des Deutschen Bundestages bekundet, sie hätten sich verpflichtet gefühlt, den europäischen Rahmenbeschluss zum Haftbefehl „eins zu eins“ in deutsches Recht zu überführen. Sie mussten sich wegen solcher Äußerung von Verfassungsrichter Udo Di Fabio belehren lassen, dass sogar ein Untätigbleiben des nationalen Gesetzgebers in der Justizpolitik von Gemeinschaftsrechts wegen nicht sanktioniert werden könne. Dazu etwa die Berichterstattung von Reinhard Müller in der FAZ Nr. 138 vom 17. Juni 2005, S. 2. 
setzgebenden Körperschaften64. Vielleicht kann diese Praxis erklären, warum die Neigung zum mitgliedstaatlichen „Aufsatteln“ so groß ist. Denn diese wahrhaft machiavellistisch anmutende Vorgehensweise mag aus politischer Sicht verständlich sein, um eigenen rechtspolitischen Vorstellungen die parlamentarische Mehrheit und vor allem freies Geleit durch den Bundesrat zu sichern. Eine solche Vorgehensweise bleibt aus gemeinschafts- wie aus verfassungsrechtlicher Sicht aber gegenüber den europäischen Institutionen ebenso wie gegenüber den gesetzgebenden Körperschaften schlicht inakzeptabel: Der Gemeinschaft wird damit die Verantwortung für eine von ihr in diesem Umfang nicht veranlasste Regelung zugeschoben, ja sie wird möglicherweise sogar zum Sündenbock für ein unpopuläres Rechtsetzungsvorhaben gemacht. Und in verfassungsrechtlich ebenso zweifelhafter Weise werden Bundestag und Bundesrat unter dem Vorwand gemeinschaftsrechtlicher Bindung fremdbestimmt, um den Erlass eines Gesetzes zu erreichen, für das eine politische Mehrheit unter Wahrung der in einer rechtsstaatlichen Demokratie selbstverständlichen Transparenzanforderungen nicht erreichbar wäre. Dass eine solche Vorgehensweise inakzeptabel ist, bedarf eigentlich keiner weiteren Ausführung. Die Gesetzgebungskultur bei der Umsetzung von Gemeinschaftsrecht bedarf offenbar dringend einer Reform an Haupt und Gliedern65.

Dieser Befund zeigt deutlich, wie segensreich die Einrichtung eines weisungsfrei gestellten Gesetzgebungs- und Beratungsdiens-

64 Im Regierungsentwurf zu diesem Gesetz (o. Fußn. 58, S. 15 f.) ist zu lesen, dass andere Umsetzungsmöglichkeiten als die im Entwurf Gewählten angesichts der detaillierten Vorgaben der Richtlinie 2003/35/EG nicht zur Verfügung gestanden hätten. Diese Behauptung trifft angesichts der Offenheit der Regelungen in der Richtlinie und in der ihr zugrunde liegenden Aarhus-Konvention ersichtlich nicht zu. Siehe zu den Umsetzungsoptionen bereits Thomas von Danwitz, AarhusKonvention: Umweltinformation, Öffentlichkeitsbeteiligung, Zugang zu den Gerichten, NVwZ 2004, S. 273 (277, 278 ff.); ebenso Matthias Schmidt-Preuß, Gegenwart und Zukunft des Verfahrensrechts, NVwZ 2005, S. 489 (494 ff.), der zu Recht von einem „Optionsmodell“ spricht.

65 Die Novellierung der Gemeinsamen Geschäftsordnung für die Bundesministerien zum 1. September 2000 kann insoweit nur als erster Schritt angesehen werden. Dazu Brigitte Zypries/Cornelia Peters, Eine neue Gemeinsame Geschäftsordnung für die Bundesministerien, ZG 2000, S. $316 \mathrm{ff}$. 
tes auf mitgliedstaatlicher Ebene insoweit wirken könnte. Die unabhängigen Fachleute sollten namentlich die Frage beantworten, welchen Umsetzungs- und Einpassungsbedarf eine Gemeinschaftsrichtlinie konkret auslöst, wie weit die gemeinschaftsrechtliche Bindung reicht und welche Umsetzungsoptionen aus gemeinschafts-, verfassungs- und fachrechtlicher Perspektive überhaupt bestehen. Eine solche Begutachtung könnte sich zudem als erheblicher Gewinn für die Transparenz demokratischer Entscheidungsfindung erweisen. Da sie den parlamentarischen Gremien überdies sehr genau vor Augen führen würde, in welchem Umfang gemeinschaftsrechtliche Bindungen $\mathrm{zu}$ beachten und mitgliedstaatliche Freiräume verblieben sind, würde sie langfristig einen fraglos wichtigen Beitrag zur verantwortungsvollen Ausübung der zwischen Gemeinschaft und Mitgliedstaaten geteilten Rechtssetzungszuständigkeiten leisten.

\section{Fazit}

Die Überlegungen haben gezeigt, dass es zweifelsohne Wege zu besserer Gesetzgebung in Europa gibt, die man beherzt beschreiten kann, ohne sich im Zwist gegenseitiger Schuldzuweisungen zu verlieren oder Maßnahmen zu betreiben, die mit dem institutionellen Gefüge von Gemeinschaft und Union a priori unvereinbar sind. Bei aller Notwendigkeit von Veränderungen im Bereich der Gemeinschaftsrechtsetzung selbst ist besonders zu betonen, dass namentlich die Mitwirkung der Mitgliedstaaten an der Gemeinschaftsrechtsetzung und vor allem ihre Verantwortung für die Umsetzungsgesetzgebung erheblich besser wahrgenommen werden müssen. Diese Einsicht bedingt vor allem, dass sich die in der Gesetzgebung Tätigen auf ihre Verantwortung für eine verbesserte Überzeugungskraft der Rechtsordnung besinnen. Denn ohne echte Verbesserungen in der Rechtsetzungsqualität kann die Befolgungsbereitschaft durch die Bürger nicht gewährleistet werden. Dieses rechtsstaatliche Grundanliegen der Suche nach Wegen zu besserer Gesetzgebung ist fraglos ohne Alternative. Daher müssen wir uns auf den Weg machen, beharrlich und ohne Verdruss, auch wenn es ein langer und mitunter steiniger Weg werden wird. 


\section{Impressum}

Herausgeber

Prof. Dr. Bernd von Hoffmann, Prof. Dr. Gerhard Robbers

Unter Mitarbeit von

Marcel Nuys und Oliver Windgätter

Redaktionelle Zuschriften

Institut für Rechtspolitik an der Universität Trier,

Im Treff 24, 54296 Trier, Tel. +49 (0)651 / 201-3443

Homepage: http://www.irp.uni-trier.de,

Kontakt: sekretariat@irp.uni-trier.de.

Die Redaktion übernimmt für unverlangt eingesandte Manuskripte keine Haftung und kann diese nicht zurückschicken. Namentlich gezeichnete Beiträge geben nicht in jedem Fall die Meinung der Herausgeber/Redaktion wieder.

Bezugsbedingungen

Die Hefte erscheinen in unregelmäßigen Abständen mehrfach jährlich und können zum Stückpreis zuzüglich Porto im Abonnement oder als Einzelheft bei der Redaktion angefordert werden. Die zur Abwicklung des Abonnements erforderlichen Daten werden nach den Bestimmungen des Bundesdatenschutzgesetzes verwaltet.

(C) Institut für Rechtspolitik an der Universität Trier, 2005 ISSN 1616-8828 